\title{
Teleatendimento no enfrentamento da COVID-19 em um município de Minas Gerais: relato de experiência de profissionais residentes
}

\author{
Tele-attendance in coping with COVID-19 in a city in Minas Gerais: experience report of \\ resident professionals
}

\section{Teleasistencia para afrontar COVID-19 en una ciudad de Minas Gerais: informe de experiencia de profesionales residentes}

Amanda Maria Batista Meneghini ${ }^{1}$, Ana Carolina Malaquias ${ }^{1}$, Andressa Castanheira Barcelos ${ }^{1}$, Cíntia Aparecida Santos ${ }^{1}$, Daniella Almeida Silva Brum¹, Dirlene Rozária Pereira ${ }^{1}$, Geisilane Nogueira da Silva ${ }^{1}$, Luiz Carlos da Silva Júnior ${ }^{1}$, Mariana Silva Guimarães de Menezes ${ }^{1}$, Marlon Willian da Silva ${ }^{1}$, Nadinara Costa Ferreira ${ }^{1}$, Sabrina Brombim Zanchetta ${ }^{1}$, Thaylla Haydée Silva Pinto $^{1}$, Elaine Cristina Dias Franco ${ }^{1 *}$, Joseane da Silva².

\section{RESUMO}

Objetivo: relatar a experiência vivenciada por profissionais residentes do Programa de Residência Multiprofissional em Saúde do Adolescente da UFSJ e Secretaria Municipal de Saúde, no cotidiano da Central de Atendimentos Alô Corona. Relato de experiência: Implantado em março de 2020, o Alô Corona tem como finalidade proporcionar informações à população, por teleatendimento, sobre diversos assuntos relacionados à COVID-19, evitando assim o deslocamento dos usuários às unidades de saúde da Atenção Primária à Saúde (APS) em um município no interior de Minas Gerais. A prática educativa desenvolvida, por profissionais residentes, via ligação telefônica, dá ao usuário a possibilidade de ter suas dúvidas resolvidas em um espaço confiável e seguro de acesso à informação, considerando que desde janeiro do corrente ano a sociedade está imersa em um contexto de excesso de informações, muitas vezes desencontradas. Considerações finais: O Alô Corona vem se mostrando importante dispositivo de promoção da saúde e prevenção dos agravos relacionados à COVID-19.

Palavras-chave: Infecções por coronavírus, Pandemias, Educação em saúde.

\begin{abstract}
Objective: to report the experience of professionals living in the Multiprofessional Residency Program in Adolescent Health at UFSJ and the Municipal Health Secretariat, in the daily routine of the Alô Corona Call Center. Experience report: Implemented in March 2020, Alô Corona aims to provide information to the population, via call center, on various subjects related to COVID-19, thus avoiding the displacement of users to primary health care $(\mathrm{PHC})$ health units in a municipality in the interior of Minas Gerais. The educational practice developed by resident professionals, via telephone call, gives the user the possibility of having their doubts resolved in a reliable and safe space of access to information, considering that since January this year, society is immersed in a context of excess of information, often mismatched. Final considerations: Alô Corona has proved to be an important device for health promotion and prevention of diseases related to COVID-19.
\end{abstract}

Keywords: Coronavirus infections, Pandemics, Health education.

1 Universidade Federal de São João del Rei (UFSJ), Divinópolis - MG. *E-mail: elainefranco@ufsj.edu.br

2 Secretaria Municipal de Saúde, Divinópolis - MG. 


\section{RESUMEN}

Objetivo: informar la experiencia de los profesionales que viven en el Programa de Residencia Multiprofesional en Salud de Adolescentes en UFSJ y la Secretaría de Salud Municipal, en la rutina diaria del Centro de Llamadas Alô Corona. Informe de experiencia: Implementado en marzo de 2020, Alô Corona tiene como objetivo brindar información a la población, a través de call center, sobre diversos temas relacionados con COVID-19, evitando así el desplazamiento de los usuarios a las unidades de salud de atención primaria (APS) de un municipio en el interior de Minas Gerais. La práctica educativa desarrollada por profesionales residentes, a través de llamadas telefónicas, brinda al usuario la posibilidad de resolver sus dudas en un espacio confiable y seguro de acceso a la información, considerando que desde enero de este año, la sociedad está inmersa en un contexto de exceso de información, a menudo no coincidente. Consideraciones finales: Alô Corona ha demostrado ser un dispositivo importante para la promoción de la salud y la prevención de enfermedades relacionadas con COVID-19.

Palabras clave: Infecciones por coronavirus, Pandemias, Educación en salud.

\section{INTRODUÇÃO}

A infecção pelo novo coronavírus SARS-COV-2 foi identificada pela primeira vez em humanos em dezembro de 2019, na cidade de Wuhan/China. O aumento exponencial de casos caracterizou a infecção inicialmente como um surto. No final de janeiro de 2020, a OMS declarou a situação como uma emergência em saúde pública de interesse internacional e em 11 de março de 2020 declarou a Covid-19 como pandêmica (WHO, 2020).

Nesse cenário, pesquisadores vêm estudando o vírus como estratégia para a definição de suas características, transmissibilidade, espectro de manifestações clínicas e potencial virulência (PERLMAN S, 2020). As vias primordiais de transmissão do COVID-19 incluem transmissão direta através de tosse, espirro e perdigotos, além de transmissão por contato com mucosa oral, nasal e dos olhos (PENG X, et al., 2020; PORTUGAL JKA, 2020). No Brasil, desde o primeiro teste positivo para COVID-19 identificado em fevereiro de 2020 observa-se um aumento significativo dos casos confirmados, já apresentando quase 3,3 milhões de casos positivos e mais de 100 mil mortes acumuladas (BRASIL, 2020a).

Com a ocorrência de transmissão comunitária, o distanciamento social e o isolamento dos casos suspeitos estão entre as medidas indicadas para mitigar o aumento rápido dos casos da doença e, consequentemente, a sobrecarga na rede de assistência à saúde (DIVINÓPOLIS, 2020; MINAS GERAIS, 2020; WHO, 2020). No entanto, tais recomendações exigem que os países sejam capazes de exercer o papel de suporte àqueles que nesse momento vivem em condições permeadas por vulnerabilidades sociais, por meio de políticas econômicas e sociais emergenciais, além de prover assistência à saúde nos diferentes níveis que compõem a rede assistencial e aquisição de conhecimento acerca da COVID-19 por meio de diferentes estratégias de comunicação com difusão de informação qualificada e confiável (SULLIVAN R e CHALKIDOU K, 2020; WHO, 2020).

Diante da gravidade da pandemia de Covid-19, as pesquisas científicas são fundamentais para a definição das informações fidedignas e do conjunto de estratégias e ações voltadas para o controle da pandemia. Contudo, a produção do conhecimento científico exige tempo e investimento de médio e longo prazo, o que traz à luz a importância de estratégias de ampliação da divulgação científica e informação qualificada para a população (FIOCRUZ, 2020).

Ter acesso à comunicação e fontes de informação confiáveis, atualizadas, relevantes e baseadas em evidências científicas é fundamental para a garantia do direito à saúde e para controle e combate da COVID-19, sendo também a maneira mais eficaz de combater desinformação e fake News (FIOCRUZ, 2020; OPAS, 2020). Nesse contexto, por intermédio das atividades de pesquisa e extensão, as Universidades desempenham papel primordial viabilizando troca de conhecimentos, descobrindo e redescobrindo soluções (SILVA MRF et al., 2020). 
Desse modo, observa-se que medidas macropolíticas, relacionadas ao acesso às políticas públicas sociais, a garantia de assistência à saúde, dentre outras, estão interligadas com ações de educação em saúde no contexto micropolítico, aqui entendido como o espaço da inter-relação que se estabelece entre o usuário e o serviço de saúde que, à luz de Merhy, configura-se como uma tecnologia leve em saúde.

Para o autor, o trabalho em saúde é movido por dispositivos, denominados de tecnologias leves, leveduras e duras que se interpenetram no cotidiano da assistência à saúde e possuem como fim comum a garantia de acesso universal à saúde (MERHY EE, 2005; SANTOS DS et al., 2018).

Em seus diversos guidelines, a OMS torna explícita a necessidade urgente de educar o público em geral sobre a COVID-19 com destaque para suas repercussões e gravidade para a saúde individual e coletiva, bem como para a importância de práticas individuais e coletivas na prevenção da propagação da doença (WHO, 2020). Nesse ínterim, as práticas educativas, vistas como tecnologias leves em saúde, mostram-se pertinentes por terem suas bases sustentadas em relações entre e com usuários associadas ao preparo desses para (con)viver com novos modos de produção do cuidar de si e do outro (GOMES LB e MERHY EE, 2011).

Novas formas de acesso às informações têm sido suscitadas, uma vez que há necessidade de manter o isolamento social, mas também garantir o acesso à informação e o acompanhamento das condições de saúde e fatores de risco para o adoecimento da população.

Diversas são as estratégias em curso no território nacional sustentadas nas premissas do Programa Telessaúde Brasil, instituído em 2007 pelo Ministério da Saúde, com o objetivo de desenvolver ações de apoio às equipes de atenção básica por meio da educação permanente à distância e que em 2020 ganhou nova roupagem ao se tornar também espaço de acompanhamento das condições de saúde, orientação e educação da população geral (BRASIL, 2011; BRASIL, 2015; MAEYAMA MA e CALVO MCM, 2018).

O termo telessaúde é entendido como a disposição de serviços realizados por profissionais da saúde que empregam as Tecnologias de Informação e Comunicação (TIC) como substituição do contato direto com o paciente, troca de dados necessários para prevenção, diagnóstico ou tratamento, assim como auxiliar na educação continuada desses profissionais e na realização de pesquisas nas quais a distância é um fator crítico (CATALANI B et al., 2016; FILHO BABS e TRITANY EF, 2020).

Ancorada nas premissas da Atenção Primária à Saúde, na necessidade de instituir novas formas de oferecer informação à população, nas medidas de mitigação da disseminação da COVID-19 e no entendimento que a educação em saúde configura-se como um dispositivo de produção do conhecimento com e pela comunidade, a Secretaria Municipal de Saúde (SEMUSA) de um município de Minas Gerais, em parceria com a Universidade Federal de São João del Rei (UFSJ), implantou em 23 de março de 2020 a Central de Atendimentos - ALÔ CORONA.

O objetivo deste manuscrito é relatar a experiência vivenciada por profissionais residentes do Programa de Residência Multiprofissional em Saúde do Adolescente (REMSA) da UFSJ no cotidiano de atendimento da Central de Atendimentos Alô Corona.

\section{RELATO DE EXPERIÊNCIA}

Em meio à pandemia da COVID-19, em março de 2020 a Secretaria Municipal de Saúde e o Programa de Residência Multiprofissional em Saúde do Adolescente, desenvolveram o projeto de assistência à saúde, via teleatendimento, denominado de Alô Corona. Esse projeto é formado por um grupo multidisciplinar de profissionais residentes, composto por enfermeiros, dentistas, nutricionistas, psicólogos, fisioterapeutas e assistentes sociais.

A gestão da equipe de residentes é feita por uma enfermeira vinculada à SEMUSA e uma docente/tutora da REMSA/UFSJ. Os atendimentos foram realizados na sede da SEMUSA de segunda a sexta-feira, de $07 \mathrm{~h} 00$ às $18 \mathrm{~h} 00$, exceto em feriados e finais de semana. 
Até a implantação da Central de Atendimentos houve reuniões de planejamento para alinhar as necessidades materiais, físicas e humanas da proposta de intervenção. Na qualificação permanente dos residentes, relacionada à COVID-19 e suas interfaces biopsicossociais, foram usados os guidelines da OMS, protocolos do Ministério da Saúde e do município, bem como os cursos ofertados pelas plataformas de educação à distância da FIOCRUZ e UNA-SUS.

A Central de Informações - Alô Corona tem como finalidade proporcionar informações à população, por teleatendimento, sobre diversos assuntos relacionados à COVID-19, evitando assim o deslocamento dos usuários às unidades de saúde daAtenção Primária à Saúde (APS) no município.

O início das atividades em 23 de março trouxe para a equipe diferentes sentimentos e expectativas, considerando as singularidades que envolvem a COVID-19. Em reunião virtual realizada ao final do primeiro dia de atividades os residentes relataram sentimentos relacionados com o medo do novo, do imprevisto ao atender uma ligação, bem como remeteram a satisfação de oferecer ao usuário informações importantes para a proteção de suas condições de saúde.

Quanto às expectativas, foram relacionadas a ofertar à população um serviço de assistência à saúde pautada em novas formas de cuidado diante do isolamento social necessário durante a pandemia, à adesão da comunidade ao serviço ofertado e à possibilidade da central ser vista como um espaço de escuta qualificada da comunidade.

Alicerçados na literatura especializada, busca-se com o Alô Corona o enfrentamento à pandemia, com práticas de educação em saúde pautadas na tríade: I) profissionais de saúde implicados com a defesa da vida e que tenham suas práticas sustentadas na prevenção da COVID-19, promoção da saúde e em práticas de cuidado humanizado; II) gestores de saúde que contribuem para execução da educação em saúde; e III) comunidade envolvida de forma autônoma nos cuidados individuais e coletivos para enfrentamento da COVID-19.

No cotidiano das atividades observou-se, no primeiro trimestre de teleatendimentos, que houve um número maior de ligações no primeiro mês. Para a equipe à divulgação do Alô Corona nas redes sociais e mídias televisas favoreceu o acesso à informação e o uso do serviço ofertado pela população. Já nos meses seguintes verificou-se que a divulgação ocorreu de forma pontual, provocando um possível distanciamento da comunidade.

Quanto aos registros dos motivos relacionados aos teleatendimentos, os residentes observaram que os contatos foram relacionados com os decretos municipais de definição de estratégias para o isolamento social, funcionamento de estabelecimentos comerciais, escolas e templos religiosos; à busca por informações sobre sinais e sintomas da COVID-19, formas de prevenção e fluxos assistencial no munícipio.

Para os residentes a diversidade de informações ofertadas no cotidiano do Alô Corona reforça a necessidade de preparo prévio para lidar com as demandas que surgem relacionadas à COVID-19, mas também aos desfechos que ela representa em diferentes seguimentos da sociedade.

Exercer o papel de ponto de referência para a população geral através do teleatendimento permitiu aos residentes observarem, no cotidiano do Alô Corona, a ocorrência de dificuldades de comunicação; divergências no entendimento dos decretos e; dificuldades de entendimento da população, muitas vezes relacionada com a qualidade do áudio das ligações.

\section{DISCUSSÃO}

O anúncio da Covid-19 como pandemia que inaugurava o ano de 2020 trouxe para os contextos sociopolítico, econômico, educacional e de assistência à saúde a necessidade de estabelecer novas rotas de garantia de direitos para a população ante as rupturas sociais advindas das medidas de controle da disseminação da doença nas diferentes esferas de governo (DIVINÓPOLIS, 2020; MINAS GERAIS, 2020; WHO, 2020). 
Neste contexto, ações de teleatendimento relacionadas ao COVID-19 têm sido adotadas no âmbito do Sistema Único de Saúde (SUS) por favorecer o isolamento domiciliar da população e evitar ao máximo o esgotamento dos serviços presenciais de saúde (BRASIL, 2020b).

A característica de multiprofissionalidade que compõe a equipe de residentes alocados no Alô Corona trouxe à proposta de intervenção a transversalidade de diferentes saberes e subjetividades, permitindo a proposição de novas modelagens para as ações educativas, via teleatendimento, com valorização do conhecimento do usuário, construção dialógica do conhecimento, bem como escuta atenta e cuidadosa para nuances que extrapolam questões biomédicas da COVID-19. O cuidado e atenção à saúde têm como baliza o trabalho "vivo em ato", que se sustenta em relações de interação e subjetividades que permeiam o profissional da saúde e a comunidade (SANTOS DS et al., 2018).

Diante disso, entende-se que a proximidade com a comunidade, por meio de divulgação do Alô Corona em mídias e redes sociais, favoreceu o reconhecimento desse espaço como um lócus de produção do cuidado, de relações confiáveis e humanas com escuta qualificada (SANTOS DS et al., 2018). Portanto, percebe-se na experiência vivenciada que a estratégia de construção de imagem social para a Central de Atendimentos deve ser adotada como um dispositivo essencial pela equipe gestora do Alô Corona.

As ligações com motivos relacionados à busca de informações sobre decretos municipais e estaduais sugere maior preocupação da população com a economia, em detrimento de conhecimentos relacionados à COVID-19, fato que pode ser justificado por estudos que demonstram impactos negativos nos setores da economia brasileira e mundial devido à pandemia (JUNIOR RRF e RITA LPS, 2020; PORSSE AA et al., 2020; SENHORAS EM, 2020).

Quanto ao número de ligações, percebe-se que a quantidade de ligações recebidas era sempre maior em dias que os decretos municipais eram publicados, já que, além de definirem ações de ordem pública, também norteiam a população geral em sua reorganização perante a nova realidade a fim de propiciar o achatamento da curva epidemiológica de transmissão (FREITAS CM, et al., 2020).

Depreende-se do cotidiano do Alô Corona que a prática educativa desenvolvida neste lócus dá ao usuário a possibilidade de ter suas dúvidas resolvidas em um espaço confiável e seguro de acesso à informação, considerando que desde janeiro do corrente ano a sociedade está imersa em um contexto de excesso de informações, muitas vezes desencontradas.

Esse conjunto de práticas de educação em saúde favorece a autonomia das pessoas, permitindo o acesso a conhecimentos que fortalecem o usuário para o posicionamento ante decisões de saúde que impactam a si mesmo e a coletividade (FERNANDES KJSS et al., 2016).

Sabe-se que o comportamento e a saúde da população podem ser influenciados de acordo com as informações incorporadas pela mesma em suas práticas cotidianas. Nesse sentido, sendo a literatura sobre o prognóstico da COVID-19 ainda escassa e transitória, há lacunas na compreensão dos indivíduos a respeito do tema, o que facilita a produção e propagação de Fake News, colocando em descrédito as medidas de proteção e segurança para o enfrentamento da doença adotadas como diretrizes no SUS (NETO M, 2020). Com isso, reforça-se a necessidade de investimentos em programas de educação em saúde (JÚNIOR JHS et al., 2020).

A experiência vivenciada pelos residentes ao propor e implantar a Central de Teleatendimento revela que o Alô Corona tem se mostrado uma importante ferramenta educativa e assistencial para a população e para apoio aos profissionais da APS. Diante das experiências vivenciadas e ajustes cotidianos, a Central de Teleatendimento vem ao longo dos últimos meses expandindo seu escopo de atuação com a produção de materiais educativos para a população geral.

Há para a equipe de trabalho e coordenação do Alô Corona o entendimento da educação em saúde, nesse caso, como um processo de (re)construção e (re)significação de diferentes saberes que compõem o cotidiano dos usuários e também dos profissionais residentes que necessitam, neste momento, de uma remodelagem para favorecer a apropriação da população das medidas necessárias ao enfrentamento do COVID-19. 


\section{REFERÊNCIAS}

1. BRASIL. Ministério da Saúde. Portaria no 2.546, de 27 de outubro de 2011. Redefine e amplia o Programa Telessaúde Brasil, que passa a ser denominado Programa Nacional Telessaúde Brasil Redes (Telessaúde Brasil Redes). Brasília: Diário Oficial da União, 2011.

2. BRASIL. Painel Coronavírus. 2020a. Disponível em: https://covid.saude.gov.br/. Acesso em: 15 Ago. 2020.

3. BRASIL. Ministério da Saúde. Secretaria de Atenção Especializada à Saúde (SAES). Departamento de Atenção Hospitalar, Domiciliar e de Urgência (DAHU).Protocolo de Manejo Clínico para o Novo Coronavírus (2019-n-Cov). Brasília: Ministério da Saúde, 2020b.

4. BRASIL. Ministério da Saúde. Secretaria de Gestão do Trabalho e Educação na Saúde. Secretaria de Atenção à Saúde. Custeio dos Núcleos de Telessaúde: Manual Instrutivo. Brasília: Ministério da Saúde, 2015.

5. CATALANI B, et al. Tele-educação e teleatendimento em disfagia orofaríngea: revisão de literatura. DistúrbComun, 2016;28(4): 638-648.

6. DIVINÓPOLIS. Prefeitura Municipal de Divinópolis. Decreto no 13.756, de 13 de abril de 2020. Declara estado de calamidade pública em todo o território do Município de Divinópolis para fins de prevenção e de enfrentamento à COVID-19 e ratifica a necessidade do cumprimento das medidas emergenciais de restrição e acessibilidade a determinados serviços e bens públicos e privados cotidianos em decorrência da pandemia, fixadas em âmbito estadual por deliberações do Comitê Extraordinário COVID-19.

7. FERNANDES KJSS, et al. Relato de experiência: vivências de extensão na comunidade. Rev. Ciênc. Ext., 2016; 12(1): 97-104.

8. FILHO BABS, TRITANY EF. COVID-19: importância das novas tecnologias para a prática de atividades físicas como estratégia de saúde pública. Cad. Saúde Pública, 2020; 36(5):e00054420.

9. FIOCRUZ. Fundação Oswaldo Cruz. Emergência da COVID-19 sinaliza importância da informação e comunicação para a saúde. 2020.

10. FREITAS CM, et al. Centro de Estudos e Pesquisas em Emergências e Desastres em Saúde. A gestão de riscos e governança na pandemia por COVID-19 no Brasil: análise dos decretos estaduais no primeiro mês. 2020.

11. GOMES LB, MERHY EE. Compreendendo a Educação Popular Em Saúde: Um Estudo Na Literatura Brasileira. Cadernos de Saúde Pública, 2011; 27 (1): 7-18.

12. GRANDI MS. Colapso e determinismo escalar em tempos pandêmicos: reflexões preliminares sobre a casa, o" isolamento social" e o déficit habitacional. Revista Tamoios, 2020; 16 (1).

13. JÚNIOR JHS, et al. Da desinformação ao caos: uma análise das Fake News frente à pandemia do Coronavírus (COVID-19) no Brasil. Cadernos de Prospecção, 2020; 13 (2): 331-346.

14. JUNIOR RRF, RITA LPS. Impactos da Covid-19 na Economia: limites, desafios e políticas. Cadernos de Prospecção, 2020; 13(2): 459-476.

15. MAEYAMA MA, CALVO MCM. A Integração do Telessaúde nas Centrais de Regulação: a Teleconsultoria como Mediadora entre a Atenção Básica e a Atenção Especializada. Revista Brasileira de Educação Médica, $2018 ; 42$ (2): 63-72.

16. MERHY EE. Saúde: a cartografia do trabalho vivo. $2^{\text {a }}$ ed. São Paulo: Hucitec, 2005.

17. MINAS GERAIS. Decreto NE 113 de 12 de março de 2020. Declara SITUAÇÃO DE EMERGÊNCIA em Saúde Pública no Estado em razão de surto de doença respiratória - 1.5.1.1.0 - Coronavírus e dispõe sobre as medidas para seu enfrentamento, previstas na Lei Federal № 13.979, de 6 de fevereiro de 2020. Diário do Executivo, Belo Horizonte: Jornal Minas Gerais, 2020; ano128, 53.

18. NETO M, et al. Fake news no cenário da pandemia de Covid-19. Cogitare Enfermagem, 2020; 25 , e72627.

19. OPAS. Organização Pan-Americana da Saúde. 2020. Pandemia de COVID-19: as estatísticas de acesso à BVS e o alcance da cooperação técnica da BIREME.

20. PERLMAN S. Another decade, another coronavirus. N Engl J Med, 2020; 382: 760-762.

21. PENG X, et al. Transmission routes of n2019-nCoV and controls in dental practice. Int J Oral Sci, $2020 ; 12$ (9).

22. PORSSE AA, et al. Impactos Econômicos da COVID-19 no Brasil. Nota Técnica NEDUR-UFPR, 2020; (1).

23. PORTUGAL JKA, et al. Percepção do impacto emocional da equipe de enfermagem diante da pandemia de COVID-19: relato de experiência. Revista Eletrônica Acervo Saúde, 2020; 46: e3794.

24. SANTOS DS, et al. Processo de trabalho na Estratégia de Saúde da Família: potencialidades da subjetividade do cuidado para reconfiguração do modelo de atenção. Ciência \& Saúde Coletiva [online], 2018; 23 (3): 861-870.

25. SENHORAS EM. Novo Coronavírus e seus impactos econômicos no mundo. Boletim de conjuntura (BOCA), 2020; $1(2): 39-42$.

26. SILVA MRF, et al. Reflexões sobre as ações extensionistas e de pesquisa no combate à COVID-19 na universidade do estado do Rio Grande do Norte. Braz. J. Hea. Rev., 2020; 3 (2): 3622-3646.

27. SULLIVAN R, CHALKIDOU K. 2020. Urgent call for an exitplan: the economic and social consequences of responses to COVID-19 pandemic. Center for Global Development.

28. WHO. World Health Organization. 2020. Coronavirus disease 2019. 